\title{
Les caractéristiques des stomates des feuilles de Ficus benjamina L. comme bioindicateurs potentiels de la qualité de l'air dans la ville d'Abidjan (Côte d'Ivoire)
}

\author{
N'guessan Achille Koffi 1, Yao Sadaiou Sabas Barima ${ }^{2}$, Djédoux Maxime Angaman ${ }^{3}$, Bini Kouamé \\ Dongui ${ }^{2}$ \\ 1 Université Nangui Abrogoua, Unité de Formation et de Recherche en Sciences et Gestion de l'Environnement, BP \\ 801 Abidjan, Côte d'Ivoire. \\ 2 Université Jean Lorougnon Guédé, Unité de Formation et de Recherche en Environnement. BP 150 Daloa, Côte \\ d'Ivoire. \\ 3 Université Jean Lorougnon Guédé, Unité de Formation et de Recherche en Agroforesterie. BP 150 Daloa, Côte \\ d'Ivoire. \\ Auteur pour la correspondance : Yao Sadaiou Sabas Barima, Adresse : BP 150 Daloa, E-mail : \\ byssabas@gmail.com, Téléphone : (+225)49648867
}

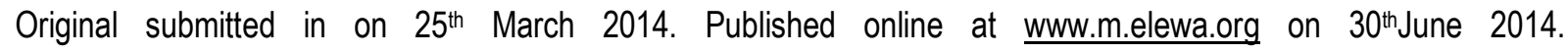
http://dx.doi.org/10.4314/jab.v78i1.12

\section{RESUME}

Objectif : L'objectif principal de cette étude est d'évaluer le potentiel des stomates de Ficus benjamina à être utilisé comme des indicateurs de la pollution locale de l'air.

Méthodologie : Des empreintes de stomates ont été prélevées sur l'espèce d'étude aux abords des axes routiers, dans des zones résidentielles et industrielles et dans des parcs. La densité, la surface des pores et la résistance des stomates ont été déterminées à partir de ces empreintes. La densité des stomates était plus élevée au niveau des classes potentiellement plus polluées (axes routiers, zones industrielles) par rapport aux classes moins polluées (zones résidentielles et parcs). Ces dernières affichent des ouvertures des pores beaucoup plus grandes dans les classes moins polluées que les classes plus polluées.

Les caractéristiques des stomates des feuilles de Ficus benjamina peuvent être de bons bio-indicateurs de la qualité de l'air. Plus précisément, la densité des stomates et la surface des pores des stomates sont potentiellement des paramètres pour évaluer la qualité de l'air.

Mots-clés : Pollution urbaine de l'air, Caractéristiques des stomates, Habitat urbain, Côte d'Ivoire

Stomatal leaf characteristics of Ficus benjamina L. as potential bioindicators of air quality in the Abidjan city (Côte d'Ivoire)

ABSTRACT

Objective: The main objective of this study is to assess the potential of Ficus benjamina stomata to be used as indicators of local air pollution.

Methodology: Stomatal prints were taken from the species of study in the vicinity of roads, in residential and industrial areas and parks. Density, pore surface and stomatal resistance were determined from these 
prints. Stomatal density was higher in the potentially most polluted classes (roads, industrial areas) compared to less polluted classes (residential areas and parks). These last classes show much larger pores in less polluted classes than most polluted classes.

Conclusion and application of results: Stomatal leaf characteristics of Ficus benjamina can be great bioindicators of air quality. Specifically, stomatal density and pore surface could be potentially parameters for assessing air quality.

Keywords: Urban air pollution, stomatal characteristics, urban habitat, Côte d'Ivoire.

\section{INTRODUCTION}

La pollution de l'environnement est l'un des problèmes majeurs dans le monde, particulièrement dans les pays en développement (Cunningham \& Berti, 1993). En effet, l'industrialisation engendre d'importantes quantités de déchets qui polluent l'environnement (khan, 2006) ces déchets représentent une menace pour la santé humaine : cancer du foie, défaillance du système respiratoire et nerveux, irritation de la peau, etc. (Tchepel \& Dias, 2011). Par ailleurs, la croissance démographique associée à l'industrialisation des villes accroissent la production de polluants à travers l'émission dans l'atmosphère des particules fines provenant des gaz d'échappement des véhicules et des cheminés des usines (Barima et al., 2014). En outre, ces polluants dans l'air sont susceptibles de contaminer l'environnement sous l'effet des facteurs influençant la pollution tels que le soleil, les précipitations, le vent et la température (Garbisu \& Alkorta, 2001 ; Atolaye et al., 2007). Les bioindicateurs, organismes biologiques fournissant des indices sur la santé des écosystèmes, ont la capacité d'accumuler les polluants dans leurs tissus (Garrec \& Van Haluwyn 2002 ; Markert et al., 2003), permettant aussi de valider sur une échelle de temps plus longue les données physicochimiques ponctuelles prises dans l'air ambiant et d'identifier la migration du polluant dans l'environnement (Fadel et al., 2008 ; Maizi et al., 2010). Ils pourront être utilisés pour déceler des changements dans l'environnement, détecter la présence de polluants, déterminer les effets de la pollution sur un écosystème et confirmer l'amélioration de la qualité de l'environnement naturel de l'organisme. Les bioindicateurs sont ainsi des mesures indirectes, substitutives, d'un phénomène écologique (Landres et al., 1988).

En Côte d'Ivoire, notamment dans la ville d'Abidjan, l'accroissement de véhicules à vieux moteurs aurait engendré une production importante de polluants de l'air. De nombreux procédés physico-chimiques ont été développés pour la surveillance de la pollution de l'air (Ali, 1993 ; Klumpp et al., 1994 ; Brighigan et al., 1997). Mais la plupart de ces méthodes sont coûteuses surtout pour les villes du Sud à faibles revenus économiques (Gombert et al. 2006). L'utilisation de procédés biologiques pour détecter et surveiller les effets de la pollution serait donc une alternative pour ces villes (Mignorance \& Rossini, 2006;Tomašević et al., 2008). Parmi ces procédés biologiques, l'utilisation des plantes comme bioindicateur de la pollution de l'air s'avère avantageuse pour évaluer la qualité de l'habitat urbain (Kardel et al., 2010). Les méthodes biologiques de détermination de la qualité de l'air ont été employées avec succès dans plusieurs pays, notamment aux Etats-Unis d'Amérique, en Allemagne, en Belgique et au Brésil en utilisant des espèces telles que Ficus religiosa et Thevetia nerifolia (Hijano et al., 2005; Verma \& Singh, 2006). La plupart de ces auteurs ont utilisé les caractéristiques biochimiques des feuilles pour l'évaluation de la qualité de l'air. D'autres comme Balassooriya et al. (2009), Kardel et al. (2010) ont utilisé les caractéristiques des stomates de Taraxacum officinalis et Plantago lanceolata pour la classification de la ville de Gent (Belgique) en zones polluées et moins polluées. A Abidjan en Côte d'Ivoire, Ficus benjamina est l'un des arbres d'ornementation les plus répandus dans la ville (Barima et al. 2014). En plus, F. benjamina est largement représentée dans toute la zone 

comme bioindicateurs potentiels de la qualité de l'air dans la ville d'Abidjan

intertropicale et les caractéristiques morphologiques de ses feuilles permettent d'y effectuer une série d'analyse. Cette espèce sera donc utilisée pour tester sa capacité à être utilisé comme un bio-indicateur de la pollution de l'air. Nous supposerons dans cette étude que la classe d'utilisation du sol influence la qualité de l'air dans laquelle cet habitat se trouve. Ainsi, du fait de la forte concentration de gaz d'échappement de véhicules à moteur et de cheminées des industries, les axes routiers et les zones industrielles seront potentiellement plus pollués que les parcs et les zones résidentielles. L'objectif principal de cette étude est d'évaluer le potentiel

\section{MATERIEL ET METHODES}

Description du site, plan d'échantillonnage et matériel biologique : La ville d'Abidjan est située au Sud de la Côte d'Ivoire, au bord du Golfe de Guinée et est comprise entre les latitudes $5^{\circ} 00^{\prime}$ et $5^{\circ} 30^{\prime} \mathrm{N}$ et les longitudes $3^{\circ} 50^{\prime}$ et $4^{\circ} 10^{\prime} \mathrm{W}$. Capitale économique de la Côte d'Ivoire, Abidjan est la ville la plus peuplée d'Afrique de l'Ouest après Lagos au Nigéria. Elle est également la deuxième plus grande ville francophone et la troisième plus grande agglomération en Afrique. Considérée comme le carrefour culturel ouest-africain voire africain, Abidjan connaît une perpétuelle croissance caractérisée par une forte industrialisation et une urbanisation galopante. Pour cette étude, la ville d'Abidjan a été subdivisé en quatre (4) classes d'utilisation du sol: zones industrielles (ZI), zones résidentielles $(Z R)$, parcs et principaux axes routiers (AR). Dans chacune de ces classes d'utilisation du sol, les échantillonnages ont été effectués sur trois sites. Sur chacun de ces sites, les prélèvements des feuilles ont été effectués à deux niveaux de hauteur: de 0 à $1,3 \mathrm{~m}(\mathrm{H} 1)$ et de 1,3 à $2,5 \mathrm{~m}(\mathrm{H} 2)$. La campagne d'échantillonnage s'est déroulée en Mars 2012 (grande saison sèche) et en Mai 2012 (grande saison de pluies). L'échantillonnage s'est effectué de 8 heures à 15 heures de la journée afin de respecter le temps d'exposition des feuilles à chaque campagne. Le matériel biologique est constitué des feuilles de $F$. benjamina récoltées sur les sites. Espèce dicotylédone de la famille des Moraceae, cet arbre a un usage généralement décoratif et s'adapte à des milieux très divers. Les feuilles sont persistantes, brillantes, coriaces et pétiolées.

Détermination des paramètres des stomates des stomates de Ficus benjamina à être utilisé comme des indicateurs de la pollution locale de l'air. Pour y arriver, les paramètres des stomates (la densité des stomates, la surface de leurs pores ainsi que la résistance des stomates) obtenus dans différentes classes d'utilisation du sol seront d'abord déterminés et comparés. Ensuite, l'influence des classes d'utilisation du sol et des saisons (sèche et pluvieuse) sur les paramètres des stomates sera mise en exergue. Enfin, la variation de ces paramètres en fonction de la hauteur de prélèvement des stomates sera déterminée.

Prélèvement des empreintes des stomates: Une feuille saine adulte de chaque plante a été choisie pour mesurer la densité des stomates, la longueur et la largeur des stomates. Sur la feuille rattachée à la plante à la hauteur $\mathrm{H} 1$ et $\mathrm{H} 2$, les faces supérieures et inférieures de celle-ci ont été nettoyées à l'eau distillée. Une partie de chaque face inférieure de la feuille a été recouverte par une mince couche de vernis à ongles incolore, tout en évitant de recouvrir la nervure centrale et la zone de marge de la feuille. Après séchage pendant 15 à 20 minutes, le vernis a été détaché méticuleusement à l'aide d'un ruban adhésif transparent puis collé sur une face propre de lame de microscopie étiquetée. Pour chacune des feuilles, trois empreintes de stomates (ou préparations) ont ainsi été récoltées.

Observations microscopiques: Au laboratoire, chacune des lames de microscope a été observée avec un grossissement de $40 \times 10$ au microscope optique (Olympus CX31) relié à une caméra (LC20). Le nombre de stomates a été compté, ainsi que la longueur et la largeur ont été mesurées. Une coupe transversale de la feuille a été faite pour nous permettre de déterminer la profondeur du stomate au microscope. Sur chaque préparation, le nombre moyen de stomates par préparation a été obtenu après 10 observations, soit un total de 90 observations pour chacun des pieds de $F$. benjamina. Les moyennes des longueurs et des largeurs des pores de stomates ont été obtenues après des mesures réalisées sur 16 stomates par préparation soit 144 mesures pour chaque pied de $F$. benjamina. Au total 216 préparations ont été faites sur l'espèce sur l'ensemble de nos sites d'échantillonnage. 

comme bioindicateurs potentiels de la qualité de l'air dans la ville d'Abidjan

Traitement des données : Plusieurs indices ont été calculés pour analyser les paramètres des stomates des feuilles en fonction de la classe d'utilisation du sol et de la hauteur de prélèvement. II s'agit de la résistance des stomates (RS), la surface des pores des stomates (SPS) et la densité des stomates (DS). La densité des stomates, définie comme le nombre de stomates par $\mathrm{mm}^{2}$ de surface foliaire (Chen et al., 2001) varie largement entre 5 et 1000 stomates par $\mathrm{mm}^{2}$ d'épiderme (Hetherington \& Woodward, 2003). La densité des stomates augmente avec l'intensité de la pollution de l'air en indiquant les zones les plus polluées et les zones les moins polluées (Woodward et al., 1995). La résistance des stomates (RS) exprime la mesure de l'inhibition de la diffusion des gaz à travers les stomates (Balasooriya et al., 2009). En cas de pollution de l'air, la limitation de la diffusion des gaz est observée en raison d'une augmentation de la (RS) (Verma \& Singh, 2006 ; Balasooriya et al., 2009). Elle est calculée pour mettre en évidence l'état de stress de la plante. RS $\left(S \cdot \mathrm{m}^{-1}\right)$ est obtenu suivant l'équation 1 :

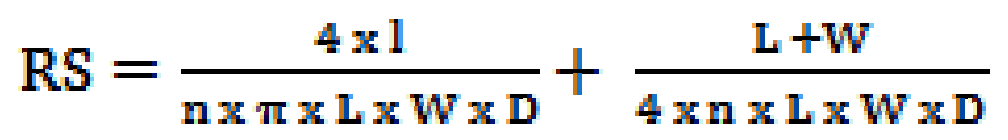

Où

$\mathrm{I}=$ profondeur du stomate $(\mathrm{m})$,

$D=$ coefficient de diffusion de la vapeur d'eau dans l'air

$\left(24,2 \times 10^{-6} \mathrm{~m}^{2} . \mathrm{S}^{-1}\right.$ à $\left.20^{\circ} \mathrm{C}\right)$,

$\mathrm{n}=$ nombre de stomates par $\mathrm{m}^{-2}$,

$W=$ largeur de l'ouverture du stomate $(m)$ et

$L=$ longueur de l'ouverture du stomate $(\mathrm{m})$.

La surface des pores des stomates (SPS) est obtenue à partir de la longueur et de la largeur de l'ouverture des stomates. Elle est calculée pour connaître le degré d'ouverture des stomates. La surface des pores des stomates est calculée à partir de l'équation (2) :

$$
\mathrm{SPS}=\frac{\mathrm{L} \times \mathrm{W} \times \pi}{4}
$$

\section{RESULTATS}

Variation de la densité des stomates entre les classes d'utilisation du sol : La densité des stomates de $F$. benjamina obtenue dans les différentes classes d'utilisation du sol (Figure 1) montre que quelques soit la saison de prélèvement, les valeurs plus élevées ont été obtenues dans les classes potentiellement plus polluées (AR et $\mathrm{ZI}$ ) et les moins élevées dans les classes moins polluées (ZR et Parcs). Relativement à la comparaison des saisons entre les classes
SPS est communément exprimé en $\mu m^{2}$.

Analyse statistiques des résultats : La normalité des données a été vérifiée à l'aide du test de Kolmogorov Smirnov. Une ANOVA à un facteur a été employée pour comparer la densité, la surface des pores et la résistance des stomates dans les quatre classes d'utilisation du sol au cours de la même période de prélèvement. Le test $t$ de Student des échantillons appariés a permis de comparer les paramètres de stomates entre les mois de Mars et de Mai pour une même classe d'utilisation du sol. L'ensemble de ces tests a été effectué à l'aide du programme Statistica 7.1 (StatSoft.Inc.1984-2005).

d'utilisation du sol, les résultats de l'analyse statistique ne révèlent pas de différence significative entre les valeurs de la densité des stomates dans les classes d'utilisation du sol AR, Zl et ZR. Par contre, l'effet saison se fait ressentir dans les parcs où la densité des stomates est significativement plus élevées en saison sèche qu'en saison des pluies (Test $t$ de Student: $p<0,05)$. 


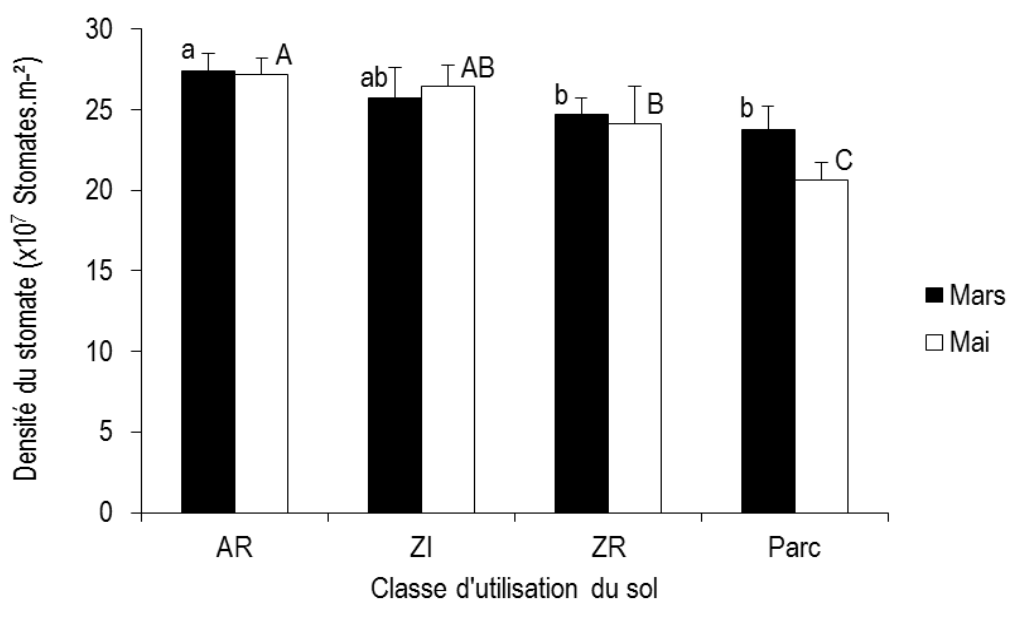

Figure 1 : Densité des stomates des feuilles de Ficus benjamina suivant les classes d'utilisation du sol pendant les mois de Mars (saison sèche) et de Mai (saison pluvieuse). AR = axe routier, $\mathrm{ZI}=$ zone industrielle, $\mathrm{ZR}=$ zone résidentielle. Les barres au-dessus des histogrammes sont les écart-types. Les lettres au-dessus des histogrammes indiquent les différences significatives entre les classes d'utilisation du sol pour le mois concerné. Seuil de significativité $\alpha=5 \%$.

Densité des stomates à différentes hauteurs: Le tableau 1 présente la variation de la densité des stomates de Ficus benjamina dans les différentes classes d'utilisation du sol en fonction des hauteurs de prélèvements et des saisons. La densité des stomates de la première hauteur de prélèvement $(\mathrm{H} 1$, correspondant à 0-1,3 m) et pour des hauteurs plus élevées $(\mathrm{H} 2$, correspondant à 1,3-2,5 $\mathrm{m})$ présente des valeurs élevées dans AR et faibles dans les parcs et ce, quelle que soit la saison de prélèvement. Dans l'ensemble, quelle que soit la saison de prélèvement, les valeurs de la densité des stomates aux hauteurs $\mathrm{H} 1$ et $\mathrm{H} 2$, ne présentent pas de différence significative dans les classes d'utilisation du sol (Test $t$ de Student : $p>0,05)$. Cependant, elle est plus élevée en AR et ZI, qu'en ZR et Parc.

Tableau 1: Densité des stomates $\left(\mathrm{x} 10^{7}\right)$ stomates $/ \mathrm{m}^{2}$ de Ficus benjamina en fonction des hauteurs de prélèvement et des classes d'utilisation du sol.

\begin{tabular}{llll}
\hline & & $\mathrm{H} 1$ & $\mathrm{H} 2$ \\
\hline AR & Mars & 26,82 & 27,06 \\
& Mai & 27,13 & 27,21 \\
ZI & Mars & 26,86 & $24,50 \mathrm{~B}$ \\
& Mai & 25,65 & $26,81^{A}$ \\
$\mathrm{ZR}$ & Mars & 24,35 & 24,35 \\
& Mai & 24,83 & 23,48 \\
Parc & Mars & 23,93 & 23,87 \\
& Mai & 26,31 & 25,42 \\
\hline
\end{tabular}

$\mathrm{AR}=$ axe routier, $\mathrm{ZI}=$ zone industrielle, $\mathrm{ZR}=$ zone résidentielle, $\mathrm{A}$ et $\mathrm{B}$ désignent les différences significatives entre les mois de Mars et Mai pour la hauteur $\mathrm{H} 2$ dans la classe d'utilisation du sol Zl, $\mathrm{H} 1=[0-1,3 \mathrm{~m}$ [, H2 = [1,3-2,5 m [. En gras, valeur significative au seuil $a=5 \%$.

Surface des pores des stomates entre les classes d'utilisation du sol : La figure 2 présente les valeurs de la surface des pores des stomates de F. benjamina dans les différentes classes d'utilisation du sol. Dans ces classes, la surface des pores observée présente des valeurs plus élevées dans les parcs et ZR par rapport aux Zl et AR. Les valeurs de la surface des pores des stomates sont significativement plus élevées en saison sèche qu'en saison des pluies (Test $t$ de Student : $p<0,05)$. 


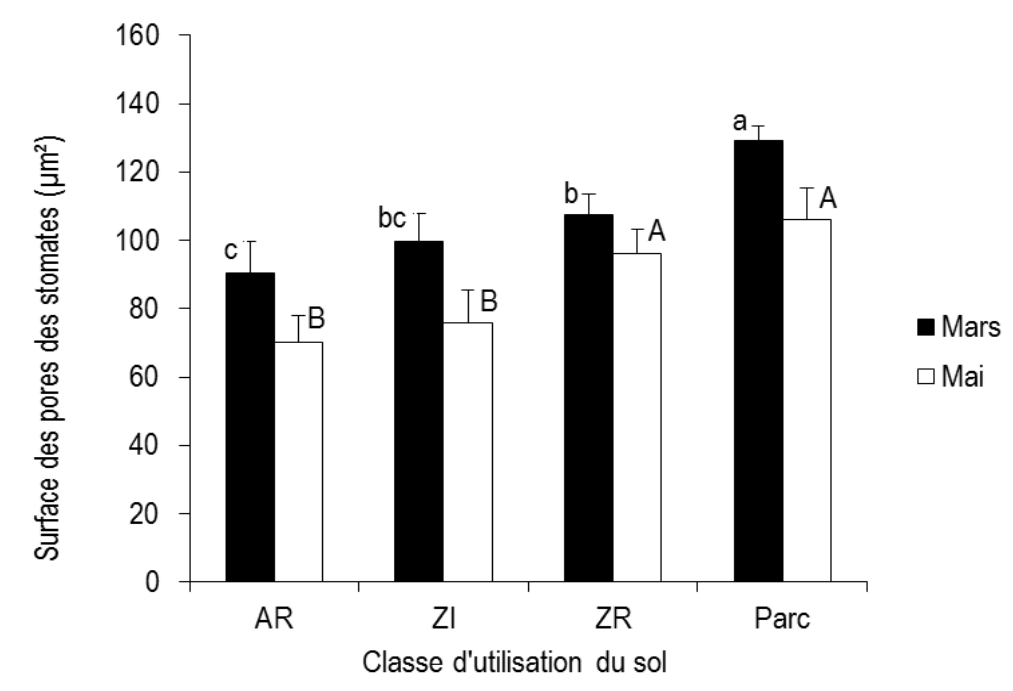

Figure 2 : Surface $\left(\mu \mathrm{m}^{2}\right)$ des pores des stomates des feuilles de Ficus benjamina suivant les classes d'utilisation du sol pendant les mois de Mars (saison sèche) et Mai (saison pluvieuse). $A R=$ axe routier, $Z \mathrm{Zl}=$ zone industrielle, $Z \mathrm{R}=$ zone résidentielle. Les barres au-dessus des histogrammes sont les écart-types. Les différentes lettres indiquent les différences significatives entre les classes d'utilisation du sol pour le mois concerné. Seuil de significativité $\alpha=5 \%$.

Surface des pores des stomates entre les hauteurs: La surface des pores des stomates de $F$. benjamina aux différentes hauteurs de prélèvement $(\mathrm{H} 1$ et H2) présente des valeurs élevées dans ZR et faibles dans $A R$, cela quel que soit le mois de prélèvement (Tableau 2). Par ailleurs, la surface des pores des stomates présente des valeurs qui varient de 64,581 $\mu \mathrm{m}^{2}$ à $121,363 \mu \mathrm{m}^{2}$, et de $67,476 \mu \mathrm{m}^{2}$ à $129,893 \mu \mathrm{m}^{2}$ respectivement pour les hauteurs $\mathrm{H} 1$ et $\mathrm{H} 2$. En outre, ces valeurs sont significativement différentes entre ces différentes hauteurs et entre les mois de prélèvement (Test t de Student : $p<0,05)$. Cependant, dans la classe Parc, elle ne présente aucune différence.

Tableau 2 : Surface $\left(\mu \mathrm{m}^{2}\right)$ des pores des stomates de Ficus benjamina en fonction des hauteurs de prélèvement des classes d'utilisation du sol.

\begin{tabular}{llll}
\hline & & H1 & H2 \\
\hline AR & Mars & $95,81^{\mathrm{b}, \mathrm{A}}$ & $112,07^{\mathrm{a}, \mathrm{A}}$ \\
& Mai & $64,58^{\mathrm{b}, \mathrm{B}}$ & $75,86^{\mathrm{a}, \mathrm{B}}$ \\
ZI & Mars & $99,31^{\mathrm{a}, \mathrm{A}}$ & $97,98^{\mathrm{b}, \mathrm{A}}$ \\
& Mai & $85,38^{\mathrm{a}, \mathrm{B}}$ & $67,47^{\mathrm{b}, \mathrm{B}}$ \\
$\mathrm{ZR}$ & Mars & $121,36^{\mathrm{b}}$ & $129,89^{\mathrm{a}, \mathrm{A}}$ \\
& Mai & 107,25 & $106,30^{\mathrm{B}}$ \\
\multirow{2}{*}{ Parc } & Mars & 83,96 & 113,43 \\
& Mai & 87,35 & 103,24 \\
\hline
\end{tabular}

$\mathrm{AR}=$ axe routier, $\mathrm{ZR}=$ zone résidentielle, $\mathrm{ZI}=$ zone industrielle, $a$ et $\mathrm{b}$ désignent les différences significatives entre les hauteurs pour le même mois, $A$ et $B$ désignent les différences significatives entre les mois pour une même hauteur, $\mathrm{H} 1=[0-1,3 \mathrm{~m}[, \mathrm{H} 2=[1,3-2,5 \mathrm{~m}[$. En gras, valeurs significatives au seuil $\mathrm{a}=5 \%$.

Résistance des stomates entre les classes d'utilisation du sol : Les valeurs de la résistance des stomates de $F$. benjamina obtenues à partir des données des différentes classes d'utilisation du sol en saisons sèche et pluvieuse sont présentées par la figure 3. Dans l'ensemble, les valeurs de la résistance des stomates les plus élevés ont été obtenues dans AR et Zl, les plus faibles sont présentes dans les parcs et $Z R$. Dans chacune des classes, les valeurs de la résistance des stomates les plus élevées sont observées pendant la saison des pluies et les plus faibles en saison sèche. Cependant, les valeurs de la 
résistance des stomates sont significativement plus élevées en saison des pluies qu'en saison sèche dans parc et ZR (Test t de Student : $p<0,05)$. Toutefois, dans les classes AR et Zl, elles ne présentent pas de différence entre les saisons.

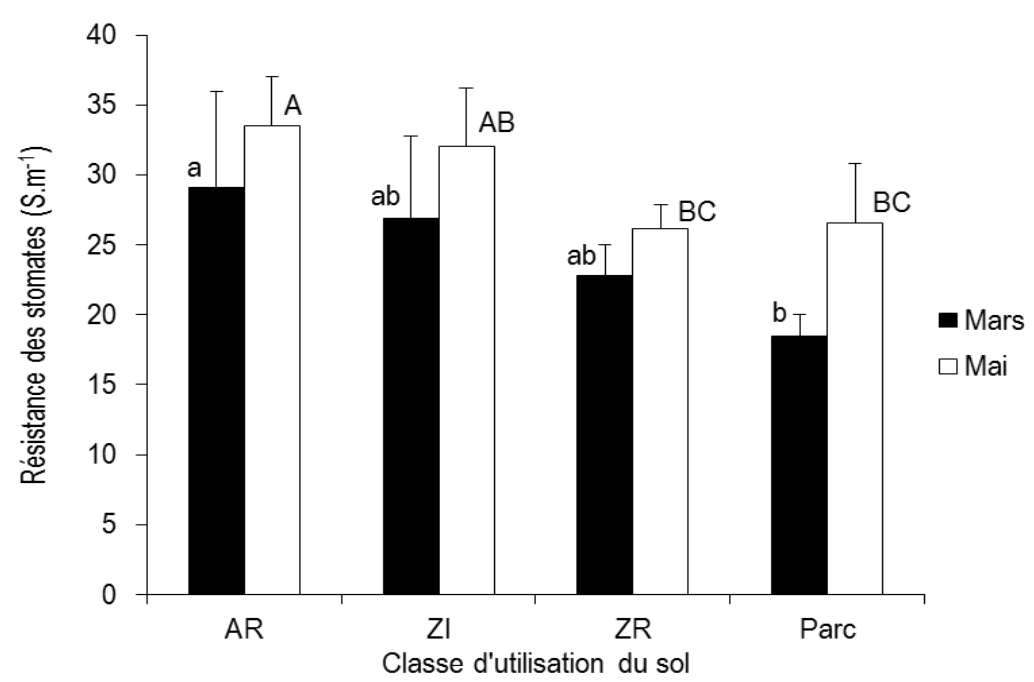

Figure 3 : Résistance $\left(\mathrm{S} . \mathrm{m}^{-1}\right)$ des stomates des feuilles de Ficus benjamina suivant les classes d'utilisation du sol pendant les mois de Mars (saison sèche) et Mai (saison pluvieuse). AR = axe routier, ZI = zone industrielle, ZR = zone résidentielle. Les barres au-dessus des histogrammes sont les écart-types. Les différentes lettres au-dessus des histogrammes indiquent les différences significatives entre les classes d'utilisation du sol pour le mois concerné. Seuil de significativité $a=5 \%$.

Résistance des stomates dans les classes d'utilisation du sol à différents niveaux de prélèvement : Le tableau 3 présente la variation de la résistance des stomates de $F$. benjamina dans les différentes classes d'utilisation du sol en fonction des hauteurs dans les mois de Mars et de Mai. Les valeurs de la résistance des stomates de la hauteur $(\mathrm{H} 1)$ varient de $22,602 \mathrm{~S} . \mathrm{m}^{-1}$ à $36,152 \mathrm{~S} . \mathrm{m}^{-1}$ et de 19,555
S. $\mathrm{m}^{-1}$ à $34,870 \mathrm{~S} \cdot \mathrm{m}^{-1}$ pour la hauteur (H2). Les valeurs les plus élevées sont obtenues aux abords des AR et les plus faibles dans les ZR. Quelle que soit la hauteur de prélèvement seules les classes AR et ZI présentent des valeurs significativement différentes, tandis que parc et ZR ne présentent pas des valeurs significatives (Test $t$ de Student : $p>0,05$ ).

Tableau 3 : Résistance des stomates $\left(\mathrm{S} . \mathrm{m}^{-1}\right)$ de Ficus benjamina en fonction des hauteurs de prélèvement et des classes d'utilisation du sol.

\begin{tabular}{llll}
\hline & & H1 & H2 \\
\hline AR & Mars & $22,60^{\mathrm{a}, \mathrm{B}}$ & $19,55^{\mathrm{b}, \mathrm{B}}$ \\
& Mai & $36,15^{\mathrm{a}, \mathrm{A}}$ & $31,66^{\mathrm{b}, \mathrm{A}}$ \\
ZI & Mars & 26,48 & $26,97^{\mathrm{B}}$ \\
& Mai & $29,83^{\mathrm{b}}$ & $34,87^{\mathrm{a}, \mathrm{A}}$ \\
$\mathrm{ZR}$ & Mars & 27,89 & 27,74 \\
& Mai & 25,84 & 27,23 \\
Parc & Mars & 29,59 & 28,53 \\
& Mai & 30,79 & 25,72 \\
\hline
\end{tabular}

$\mathrm{AR}=$ axe routier, $\mathrm{ZR}=$ zone résidentielle, $\mathrm{Zl}=$ zone industrielle, $\mathrm{a}$ et $\mathrm{b}$ désignent les différences significatives entre les hauteurs pour le même mois, $A$ et $B$ désignent les différences significatives entre les mois pour une même hauteur, $\mathrm{H} 1=[0-1,3 \mathrm{~m}[, \mathrm{H} 2=$ [ 1,3-2,5 $\mathrm{m}$ [. En gras, valeurs significatives au seuil $\alpha=5 \%$. 


\section{DISCUSSION}

La densité des stomates dans les différentes classes d'utilisation du sol durant la saison sèche et pluvieuse à différentes hauteurs présentent des valeurs significativement élevées dans les classes potentiellement plus polluées (AR et ZI) comparativement aux classes moins polluées (Parc et $Z R)(p<0,05)$. Nos résultats confirment les conclusions faites par Elagoz et al. (2006) et Balasooriya et al. (2009). Ces auteurs expliquent ces résultats par la réponse des plantes à la forte concentration de polluants dans ces milieux. En effet, les AR et les ZI sont caractérisés par leur concentration élevée en $\mathrm{O}_{3}$, $\mathrm{CO}_{2}$ sous une haute température (Elagoz et al., 2006; Pandey et al., 2007), NO et NO2 (Baldauf et al., 2013), d'une part, à l'âge et à la croissance de la plante (Woodward et al., 2002) d'autre part. Ainsi, les travaux de Barima et al. (2014) menés dans la ville d'Abidjan sur la capacité des feuilles à séquestrer les particules fines ont montré que les feuilles de Panicum maximum, Amaranthus spinosus et Ficus benjamina ont des taux de particules fines élevés $\left(45,1 \times 10^{-6} A\right)$ dans les classes $A R$ et $\mathrm{Zl}$, et faibles $\left(10 \times 10^{-6} \mathrm{~A}\right)$ dans les classes Parc et ZR. Aussi, leurs travaux montrent-ils que les classes d'utilisation les plus polluées sont les principales routes et les zones industrielles opposées aux parcs et aux zones résidentielles. Contrairement à la densité des stomates, la surface des pores des stomates présente des valeurs significativement élevées dans les classes moins polluées et faibles dans les classes polluées, $(p<0,05)$. Nos résultats confirment les conclusions faites par Kardel et al. (2010) et peuvent s'expliquer par la diminution de l'absorption des polluants, se traduisant par la réduction de la taille des stomates voire sa fermeture (Barber et al., 2004 ; Elagoz et al., 2006). Toutefois, les valeurs de la surface des pores des stomates les plus élevées sont observées en saison sèche qu'en saison des pluies. Ces observations pourraient être dues à la

\section{CONCLUSION}

L'objectif de ce travail était d'évaluer le potentiel des stomates de Ficus benjamina à être utilisé comme indicateurs de la pollution locale de l'air à partir de la densité, la surface des pores et la résistance des stomates. Après avoir vérifié les caractéristiques des stomates face aux stress environnementaux, nos résultats montrent que la densité et la résistance des stomates présentent des valeurs plus élevées dans les zones potentiellement polluées qu'en zones moins réaction de la plante à empêcher l'absorption de polluants tout en optimisant l'absorption de $\mathrm{CO}_{2}$ et en réduisant la perte d'eau due à la transpiration (Balasooriya et al., 2009). Les feuilles de F. benjamina présentent une résistance des stomates (RS) à la pollution de l'air plus importante dans les zones polluées par rapport aux zones moins polluées quel que soit la saison et la hauteur de prélèvement. Nos résultats sont en accord avec ceux de Balasooriya et al. (2009) qui ont obtenu avec Taraxacum officinalis une augmentation significative de la résistance des stomates au niveau des zones potentiellement polluées en comparaison avec les zones moins polluées $(p<0,05)$. Ce résultat s'expliquerait par le stress dû à la pollution de l'air limitant la diffusion de gaz à travers les stomates en raison d'une augmentation de RS (Verma \& Singh, 2006) et des facteurs environnementaux (Hetherington \& Woodward, 2003; Paoletti \& Grulke, 2005) comme l'humidité (86\%) qui permettrait d'une part, une dissolution des dépôts de surface et leur pénétration à travers la cuticule et d'autre part une augmentation du degré d'ouverture des stomates. Ces résultats montrent ainsi que les paramètres climatiques tels que la température, la pluviométrie, la vitesse du vent, pourraient influer sur l'action des polluants dans les feuilles (Cuny et al., 2006) entraînant une augmentation de la résistance des stomates en saison des pluies. Toutefois, faut-il noter que d'autres facteurs biotiques, liés à l'âge, au stade physiologique et à la présence de parasites pourraient déterminer la résistance, la tolérance ou la sensibilité de la plante à la pollution. Les tendances de la variation de la densité, de la surface des pores et de la résistance des stomates dans les différentes classes d'utilisation du sol obtenues avec $F$. benjamina sont similaires aux études faites par Verma \& Singh (2006) pour Thevetia nerifolia et Pal et al. (2002) pour Ficus religiosa et Bougainvillea spectabilis.

polluées. Cependant, la surface des pores des stomates présente des valeurs plus élevées en zones moins polluées qu'en zones polluées. A travers les stomates, nous avons pu vérifier la sensibilité de la plante face au stress environnemental en fonction de la saison. En somme, les caractéristiques des stomates des feuilles de Ficus benjamina peuvent être de bons bio-indicateurs de la qualité de l'air. 


\section{REMERCIEMENTS}

Cette étude a bénéficié de l'aide de la Fondation David et Alice Van Büuren (Belgique) pour l'acquisition du microscope nécessaire à l'observation et l'analyse des stomates. Nous sommes également reconnaissant au District d'Abidjan pour avoir facilité les travaux sur le terrain et au Programme d'Appui Stratégique à Recherche Scientifique (PASRES) pour les allocations reçues et l'achat des consommables. Enfin nous

\section{REFERENCES BIBLIOGRAPHIQUES}

Ali EA, 1993. Damage to plants due to industrial pollution and their use as bioindicators in Egypt. Environmental Pollution, 81: 251-255.

Atolaye B, Aremu M, 2007. Bioaccumulation of some trace elements in the body parts of fish species associated with soil sediment and water from Eoemaganiâ confluence in nasarawa state, Nigeria. Electronic Journal of Environmental, Agricultural and Food Chemistry, 6(5): 2001-2008.

Balasooriya B, Samson R, Mbikwa F, Vitharana UWA, Boeckx P, Van Meirvenne M, 2009. Biomonitoring of urban habitat quality by anatomical and chemical leaf characteristics. Environmental and Experimental Botany, 65:386-394.

Barber JL, Thomas GO, Kerstiens G, Jones KC, 2004. Current issues and uncertainties in the measurement and modelling of air-vegetation exchange and with in-plant processing of POPS. Environmental pollution, 128: 99-138.

Barima YSS, Angaman DM, N'Gouran KP, Koffi NA, Kardel F, De Cannière C, Samson R, 2014. Assessing atmospheric particulate matter distribution based on Saturation Isothermal Remanent Magnetization of herbaceous and tree leaves in a tropical urban environment. Science of the Total Environment 470: 975982.

Brighigna L, Ravanelli M, Minelli A, Ercoli L, 1997. The use of an epiphyte (Tillandsia caput-medusae morren) as bioindicator of air pollution in Costa Rica. Science of the Total Environment, 198: 175-180.

Chen LQ, Chaloner WG, Beerling DJ, Sun QG, Collinson ME, Mitchell PL, 2001. Assessing the potential for the stomatal characters of extant and fossil Ginkgo leaves to signal atmospheric $\mathrm{CO}_{2}$ change. American Journal of Botany, 88: 1309-1315. exprimons notre reconnaissance au Département de Bioingénieurs de l'Université d'Anvers (Belgique) en particulier au Professeur Roeland Samson. Le deuxième auteur est bénéficiaire d'une bourse de mobilité de la Politique fédérale belge, co-financée par les Actions Marie Curie de la Commission européenne (BELSPO).

Cunningham SD, Berti WR, 1993. Remediation of contaminated soil with green plants: An overview. In vitro Cellular and Developmental Biology, 29: 207-212.

Cuny D, Rzepka MA, Bulteau G, Lakel A, Devred I, Van Haluwyn C, 2006. Quels rôles les plantes peuvent-elles jouer vis à vis de la pollution à l'intérieur des locaux? Air Pur, 69: 33-36.

Elagoz V, Han SS, Manning WJ, 2006. Acquired changes in stomatal characteristics in response to ozone during plant growth and leaf development of bush beans (Phaseolus vulgaris L.) indicate phenotypic plasticity. Environmental pollution 140: 395-405.

Fadel D, Djamai R, Laifa A, 2008. Estimation de la pollution atmosphérique hydrocarbonée issue de la circulation automobile dans la ville de Skikda (Algérie) à l'aide de transplants lichéniques. Ghardaïa, Algeria 16-18 February 2009, 169.

Garbisu C, Alkorta D, 2001. Phytoextraction: a costeffective plant-based technology for the removal of metals from the environment. Bioressource Technology, 77: 229-236.

Garrec J.P, Van Haluwyn C, 2002. Biosurveillance végétale de la qualité de l'air : concepts, méthodes et applications/préf. Bernard Festy. Paris : Tec et Doc, 117.

Gombert S, Asta J, Seaward MRD, 2006. Lichens and tobacco plants as complementary biomonitors of air pollution in the Grenoble area (Isère, southeast France). Ecological Indicators, 6: 429-443.

Hetherington AM, Woodward $\mathrm{FI}, 2003$. The role of stomata in sensing and driving environmental change. Nature, 424: 901-908.

Hijano CF, Dominguez MDP, Gimenez RG, Sanchez $\mathrm{PH}$, Garcia I, 2005. Higher plants as bioindicators of sulphur dioxide emissions in 
urban environments. Environmental Monitoring and Assessment, 111:75-88.

Kardel F, Wuyts K, Babanezhad M, Vitharana UWA, Wuytack T, Potters G, Samson R, 2010. Assessing urban habitat quality based on specific leaf area and stomatal characteristics of Plantago lanceolata L. Environmental Pollution. Environmental Pollution, 158: 788794.

Khan AG, 2006. Mycorrhizoremediation-an enhanced form of phytoremediation. Journal of Zhejiang University Science, 7(7): 503-514.

Klumpp A, Klumpp G, Domingos M, 1994. Plants as bioindicators of air pollution at the Serra do Mar near the industrial complex of Cubatão, Brazil. Environmental Pollution, 85:109-116.

Landres PB, Verner J, Thomas, J W 1988. Ecological uses of vertebrate indicator species: a critique. Conservation biology, 2(4), 316-328.

Maizi N, Alioua A, Tahar A, Semadi F, Fadel D, 2010. The use of inferior plants as bioindicators of automobile lead pollution in the area of Annaba (Algeria)(Utilisation des végétaux inférieurs comme bio indicateurs de la pollution plombique d'origine automobile dans la région de Annaba (Algérie)). Journal of Materials and Environmental Science, 1: 251266.

Markert BA, Breure AM, Zeichmeister HG, 2003. Bioindicators \& biomonitors : principles, concepts, and applications. Elsevier. Amsterdam. 997.

Pal A, Kulshreshtha K, Ahmad KJ, Behl HM, 2002. Do leaf surface characters play a role in plant resistance to auto-exhaust pollution? Flora, 197: 47-55.

Pandey R, Chacko PM, Choudhary ML, Prasad KV, Pal $M, 2007$. Higher than optimum temperature under enrichment influences stomata anatomical characters in rose (Rosa hybrida). Scientia Horticulturae, 113: 74-81.

Paoletti E, Grulke NE, 2005. Does living in elevated $\mathrm{CO}_{2}$ ameliorate tree response to ozone? A review on stomatal responses. Environmental Pollution, 137: 483-493.

Tchepel O, Dias D, 2011. Quantification of health benefits related with reduction of atmospheric PM10 levels: implementation of population mobility approach. International Journal of Environmental Health Research, 21: 189-200.
Tomašević $M$, Vukmirović $Z$, Rajšić $S$, Tasić $M$, Stevanović B, 2008. Contribution to biomonitoring of some trace metals by deciduous tree leaves in urban areas. Environ Monit Assess, 137: 393-401.

Verma A, Singh S, 2006. Biochemical and ultrastructural changes in plant foliage exposed to auto-pollution. Environmental Monitoring and Assessment, 120: 585-602.

Woodward FI, Lake JA, Quick WP, 2002. Stomatal development and $\mathrm{CO}_{2}$ : ecological consequences. New Phytologist, 153: 477484.

Woodward FI, Kelly CK, 1995. The influence of $\mathrm{CO}_{2}$ concentration on stomatal density. New Phytologist, 131: 311-327. 